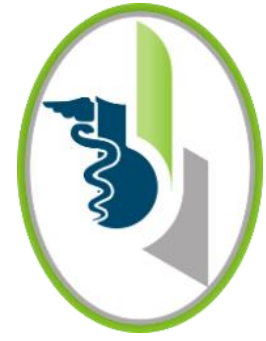

ACCESO $\odot$ ABIERTO

Para citaciones: Oyola, A., Martínez, M., Almanza, A., Trespalacios, A., Rodríguez, T., Dueñas, C. (2021). Fenómeno de lázaro: retorno de la circulación espontánea después del cese de reanimación cardiopulmonar prolongada en paciente con covid-19. Revista Ciencias Biomédicas, 10(3), 207-212. https://doi.org/10.32997/rcb-2021-3333

Recibido: 27 de abril de 2021

Aprobado: 18 de junio de 2021

Autor de correspondencia:

María Cristina Martínez Ávila cristina.martinezavila@gmail.com

Editor: Inés Benedetti. Universidad de Cartagena-Colombia.

Copyright: (C) 2021. Oyola, A., Martínez, M., Almanza, A., Trespalacios, A., Rodríguez, T., Dueñas, C. Este es un artículo de acceso abierto, distribuido bajo los términos de la licencia https://creativecommons.org/licenses/by-nc-

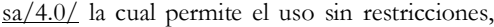
distribución y reproducción en cualquier medio, siempre y cuando el original, el autor y la fuente sean acreditados.

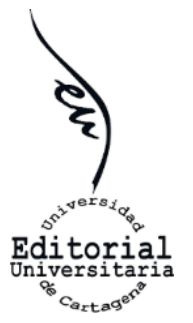

\section{Fenómeno de Lázaro: retorno de la circulación espontánea después del cese de reanimación cardiopulmonar prolongada en paciente con covid-19}

\section{Lazarus phenomenon: return of spontaneous circulation after cessation of prolonged cardiopulmonary resuscitation in a patient with covid-19}

Antonio José Oyola Yepes ${ }^{1}$ (D) , María Cristina Martínez Ávila ${ }^{2}$, Amilkar José Almanza Hurtado $^{3}$ (D), Alvano David Trespalacios Sierra ${ }^{4}$, Tomás Rodríguez Yánez ${ }^{5}$, Carmelo Dueñas-Castell ${ }^{6}$ (iD)

\footnotetext{
${ }^{1}$ Coordinación Unidad de Cuidados Intensivos, Nuevo Hospital Bocagrande, Cartagena, Colombia.

${ }^{2}$ Unidad de Cuidados Intensivos, Nuevo Hospital Bocagrande, Cartagena, Colombia.

${ }^{3}$ Especialización en Medicina Crítica y Cuidados Intensivos, Facultad de Medicina, Universidad de Cartagena, Cartagena, Colombia.

${ }^{4}$ Unidad de Cuidados Intensivos, Nuevo Hospital Bocagrande, Cartagena, Colombia.

${ }^{5}$ Especialización en Medicina Crítica y Cuidados Intensivos, Facultad de Medicina, Universidad de Cartagena, Cartagena, Colombia.

${ }^{6}$ Unidad de Cuidados Intensivos, Nuevo Hospital Bocagrande, Programa de Especialización en Medicina Crítica y Cuidados Intensivos, Facultad de Medicina, Universidad de Cartagena, Cartagena, Colombia.
}

\section{RESUMEN}

Introducción: la pandemia por la infección del SARS-CoV-2 o COVID-19 ha impactado de manera inimaginable los sistemas de salud a nivel mundial. La parada cardiorrespiratoria sigue siendo una emergencia médica potencialmente reversible que requiere la realización de un conjunto de maniobras destinadas a sustituir y restaurar la respiración y la circulación espontánea. Suspender una reanimación cardiopulmonar corresponde usualmente a un dilema ético-clínico que debe asumir el profesional de salud a cargo. El "fenómeno de Lázaro" es un síndrome inusual y de difícil explicación fisiopatológica definido como el retorno espontáneo de la circulación en ausencia de cualquier técnica de soporte vital o tras el cese de las maniobras de reanimación cardiopulmonar fallidas.

Caso clínico: se presenta el caso de una paciente de 79 años hospitalizada en unidad de cuidados intensivos por choque séptico de origen pulmonar asociado a infección por COVID-19 quien presenta parada cardiorrespiratoria que requirió maniobras de reanimación durante 40 minutos no exitosas, declarada fallecida. Tras 20 minutos del fallecimiento presenta retorno a la circulación espontánea.

Conclusión: los cambios fisiopatológicos del fenómeno de Lázaro nos recuerdan las limitaciones que tenemos para determinar cuándo finalizar la reanimación cardiopulmonar y su interrupción debe abordarse con más cuidado, especialmente en el marco de la pandemia de COVID-19.

Palabras Clave: resucitación cardiopulmonar; cuidados críticos; fenómeno lázaro; autoresucitación. 


\begin{abstract}
Introduction: the pandemic caused by the SARS-CoV-2 or COVID-19 infection has had an unimaginable impact on health systems worldwide. Cardiorespiratory arrest continues to be a potentially reversible medical emergency that requires a series of maneuvers to be performed to replace and restore spontaneous breathing and circulation. Suspending cardiopulmonar reanimation usually corresponds to an ethical-clinical dilemma that the health professional in charge must assume. The "Lazarus phenomenon" is an unusual syndrome with a difficult pathophysiological explanation, defined as the spontaneous return of circulation in the absence of any life support technique or after the cessation of failed cardiopulmonar reanimation maneuvers.
\end{abstract}

Clinic case: the case of a 79-year-old patient hospitalized in the intensive care unit for septic shock of pulmonary origin associated with COVID-19 infection is presented, who presented cardiorespiratory arrest that required unsuccessful resuscitation maneuvers for 40 minutes, declared deceased. After 20 minutes of death, she presents a return to spontaneous circulation.

Conclusions: the pathophysiological changes of the Lazarus phenomenon remind us of the limitations we have in determining when to end cardiopulmonary resuscitation and its interruption must be approached with more care, especially in the context of the COVID-19 pandemic.

Keywords: cardiopulmonary resuscitation; critical care; lazarus phenomenon; autoresuscitation.

\section{INTRODUCCIÓN}

La pandemia ocasionada por la infección por SARSCov2 o COVID-19 ha impactado de manera inimaginable los sistemas de salud a nivel mundial, generando modificaciones en los estilos de vida del ser humano y así mismo en los protocolos habituales de atención médica. A la fecha, Marzo 16 2021, se reportan en el mundo 148.015.358 confirmados y 3.138.720 muertes. En Colombia 2.795.111 casos y 72.247 muerte(1). La parada cardiorrespiratoria sigue siendo una emergencia médica potencialmente reversible que requiere la realización de un conjunto de maniobras destinadas a sustituir y restaurar la respiración y la circulación espontáneas conocidas como reanimación cardiopulmonar (RCP) (2). Ante un paciente COVID positivo surgen de manera natural las siguientes dudas ¿Qué hacemos si un paciente COVID positivo presenta un paro cardiorrespiratorio y hay que iniciar RCP?, ¿Por cuánto tiempo lo hacemos considerando su pronóstico y la escasez de recursos?.
Suspender una RCP corresponde usualmente a un dilema ético-clínico que debe asumir el profesional de salud a cargo (3). De acuerdo con la guía de orientación provisional para soporte vital básico y avanzado en adultos, niños y neonatos con COVID19 sospechoso o confirmado (3), tener una persona con un ritmo de paro durante un tiempo mayor a 10 minutos recibiendo medidas de soporte vital avanzado con vía aérea protegida y sin retorno a la circulación espontánea (RCE) es motivo suficiente para detener la RCP y declarar el fallecimiento. sin embargo, teniendo en cuenta este contexto aun cuando se desconocen cifras exactas y resultados de la RCP relacionados a COVID-19, dar cese de los esfuerzos terapéuticos supone una decisión aún más difícil de tomar; a pesar de ser este un evento frecuente en el ámbito intrahospitalario principalmente en las unidades de cuidado crítico. El "fenómeno de Lázaro", hace referencia a la famosa figura bíblica a quien Jesús resucitó: Entonces Jesús gritó: «¡Lázaro, sal de ahí!». Y el muerto salió de la tumba. Juan 11:43-44. 
Este síndrome inusual y de difícil explicación fisiopatológica se define como el retorno espontáneo de la circulación, bien sea en ausencia de cualquier técnica de soporte vital o tras el cese de las maniobras de RCP fallidas (4). Los cambios fisiopatológicos resultantes en estos fenómenos sirven como un recordatorio de nuestras limitaciones para determinar cuándo finalizar la reanimación cardiopulmonar y su interrupción debe abordarse con más cuidado.

\section{CASO CLÍNICO}

Paciente femenina de 79 años con antecedentes de hipertensión arterial quien ingresa al servicio de urgencias por cuadro de 5 días de evolución consistente en picos febriles no cuantificados, astenia, adinamia que se agudiza acompañado de dificultad respiratoria. A su ingreso se encuentra en mal estado general, presión arterial media $59 \mathrm{mmHg}$, frecuencia cardíaca $110 \mathrm{lpm}$, frecuencia respiratoria de $30 \mathrm{rpm}$, deshidratada, saturando $77 \%$ con oxígeno alto flujo, tirajes subcostales. En los paraclínicos iniciales se evidencia: leucocitosis (27080), neutrófilos (26504), linfocitos (574); potasio (6.5), creatinina (3.54); bun (86); LDH (1020); gases arteriales en equilibrio ácido base con hipoxemia severa (PaFi (PaO2/FiO2): 56.9) y tomografía de tórax con compromiso del $75 \%$ del parénquima pulmonar con patrón de vidrio esmerilado. Se decide realizar aseguramiento de la vía aérea y trasladar a unidad de cuidados intensivos COVID-19 ante sospecha clínica y radiológica de infección por SARS-COV2 y sobreinfección bacteriana con choque séptico de origen pulmonar y lesión renal aguda asociada. Se solicita RT-PCR para COVID-19 y marcadores de mal pronóstico y severidad; se inicia cubrimiento antibiótico de amplio espectro, previa toma de cultivos, corticoesteroides endovenosos de acuerdo a evidencia disponible, antiparasitario por riesgo de migración larvaria ante uso de esteroides sistémicos, gastroprotección y tromboprofilaxis.

Durante estancia en la unidad de cuidados intensivos, se confirma por RT-PCR infección por COVID-19, la paciente presenta evolución tórpida con persistencia de insuficiencia respiratoria hipoxémica severa a pesar de ventilación mecánica invasiva con miorrelajantes, sedación dual y posición prono, con requerimiento de doble soporte vasoactivo, acidosis mixta e hiperlactacidemia, oligoanuria, con disociación bun/creatinina, febril, tiempos de coagulación prolongados $\mathrm{y}$ biomarcadores de severidad alterados. Al séptimo día se evidencia bradicardia extrema que progresa hasta asistolia, constatándose ausencia de pulso, línea isoeléctrica en el visoscopio y se determina paciente en código azul.

Se inician maniobras de RCP siendo el contacto protegido según protocolo con RCE tras 10 minutos de reanimación por lo que se indicó dejar un tercer medicamento inotrópico en infusión. Pasados 15 minutos del estado posreanimación, la paciente torna en secuencia de aparición de ritmos de paro no desfibrilables que requirió nuevamente RCP de manera continua. Tras 40 minutos de asistencia y en ritmo final de asistolia, cesaron las maniobras de resucitación y se declara finalmente el fallecimiento.

Teniendo en cuenta situación epidemiológica, según las guías del Ministerio de Salud Colombiano en cuanto al manejo de cadáveres por la pandemia del COVID-19, se establece que el cadáver debe mantenerse íntegro y limitar su manipulación, sin retirar catéteres, sondas o tubos que puedan contener los fluidos del cadáver (5). La paciente continuaba con accesos vasculares, tubo orotraqueal y sonda vesical; pasados 20 minutos de declarado el deceso a la espera de las sábanas para amortajar y algodones para el cubrimiento de orificios naturales, la paciente recuperó pulso y respiración de manera espontánea evidenciada por expansión torácica. Se conectaron los monitores evidenciando frecuencia cardíaca de 100 lpm, presión arterial 123/84 mmHg, saturación de oxígeno $97 \%$ con adecuado acople al ventilador. Se solicitaron paraclínicos, gasometría arterial y se iniciaron cuidados postparada cardíaca. Finalmente, falleció 21 días después por síndrome de disfunción orgánica múltiple, choque séptico de origen pulmonar dada por neumonía viral confirmada por COVID-19. 


\section{DISCUSIÓN}

El fenómeno de lázaro se trata de una patología excepcional de difícil explicación e incluso catalogada como anecdótica, que fue reportado por primera vez en la literatura hacía el año 1982, sin embargo, no fue hasta el año 1993 que el médico anestesiólogo Jack Bray acuñó el término (6); el cual viene de la palabra Eleazaros que significa en hebreo "Dios ha ayudado" haciendo alusión al pasaje bíblico donde Jesús tras cuatro días de fallecimiento resucitó a Lázaro. Aunque es un síndrome poco conocido que puede ser desconcertante para el proveedor de atención médica, es más frecuente de lo que se cree. En las últimas décadas tanto la literatura médica, como medios de comunicación, han dejado en manifiesto más de 60 casos de autorresucitación o recuperación de la circulación espontánea tras el cese de maniobras de RCP o en ausencia de soportes vitales (7-9). La baja publicación científica de casos clínicos puede estar asociada a temores del personal de salud relacionados con acciones ético-legales, incredulidad del resto del personal asistencial ante lo ocurrido y descrédito profesional (10-11).

El mecanismo fisiopatológico exacto que produce el retorno tardío de la circulación espontánea aún es desconocido, se considera incluso que sea multifactorial. Dentro de las diversas teorías planteadas se encuentran la acidosis y trastornos hidroelectrolíticos como la hiperkalemia debido a la persistencia de potasio a nivel intracelular haciendo que el miocardio se retraiga durante largos períodos de tiempo (12), asistolia temporal transitoria después de la desfibrilación, o acción farmacológica retardada de los medicamentos usados durante la RCP como es el caso de la adrenalina (13-14). Otra hipótesis planteada, es el proceso de hiperinsuflación dinámica de los pulmones debido al exceso de volúmenes corrientes o aumento rápido de la presión positiva sin tiempo adecuado para la exhalación en la ventilación artificial generando la afectación hemodinámica (15). Es decir el aumento de la presión intratorácica puede haber conducido a atrapamiento aéreo con elevación de la presión al final de la espiración-conocida como auto-PEEPque llevó a una impedancia significativa al retorno venoso afectando el gasto cardíaco llevando a la parada cardíaca (16).

Consideramos que de acuerdo a la historia clínica de la paciente cuya principal afección era de etiología respiratoria esta hipótesis es la más acertada. Una vez se suspendió la RCP y ventilación mecánica invasiva artificial suplementaria, gradualmente las presiones torácicas a nivel del sistema respiratorio se fueron normalizando permitiendo el RCE y reaparición del pulso cardíaco. En el caso de nuestra paciente es una explicación plausible, ya que tenía requerimientos de altos volúmenes debido a la persistencia de insuficiencia respiratoria hipoxémica severa y asociado a ello presentó un ritmo de paro no desfibrilable compatible con lo descrito en la literatura. Además, habiendo recibido un aporte al $100 \%$ de oxígeno durante la RCP pudo haber sido suficiente reserva de oxígeno en el volumen residual de los pulmones para soportar sus demandas de oxígeno durante el período que no recibió asistencia. Es recomendable utilizar en lo posible ventilación mecánica protectora con el objetivo de evitar la aparición de auto-PEEP y afectar la circulación sistémica.

En cuanto al intervalo de tiempo desde el cese de $\mathrm{RCP} /$ diagnóstico de la muerte hasta el RCE, en una revisión sistemática donde se analizaron 38 fuentes bibliográficas refieren que varió entre "algunos segundos" y un máximo de 33 minutos, es de mencionar que la expresión "algunos segundos" se interpretó como un tiempo menor a 10 minutos; sin embargo en un caso descrito se encontró al paciente vivo en la morgue y en 7 casos no se contaba con la información del tiempo transcurrido (9).

No existe evidencia disponible que relacione la patología de base del paciente con la aparición del fenómeno de Lázaro. La causa de una parada cardíaca en pacientes COVID-19 está relacionada con el síndrome de distress respiratorio agudo severo que conlleva a hipoxemia refractaria así como complicaciones cardiovasculares mayores dadas por la tormenta de citocinas o la disfunción miocárdica por efecto directo del coronavirus del síndrome respiratorio agudo severo en el corazón (17). El 
pronóstico de sobrevida en PCR en pacientes COVID-19 es desconocido (18). Las Academias Nacionales de Ciencias, Ingeniería y Medicina de los Estados Unidos afirman que los estándares de atención en caso de crisis como lo es la infección por COVID-19 tienen como objetivo "salvar la mayor cantidad de vidas posibles" bajo severas limitaciones de recursos; manteniendo los principios éticos fundamentales: "justicia, cuidar, no hacer daño, uso razonable de recursos, transparencia en la toma de decisiones, coherencia, proporcionalidad y rendición de cuentas" (19) Es por esto que una vez establecido un código azul se inicia la RCP esperando el RCE.

Hay poca información en la literatura en relación a desenlaces post RCP en COVID. Se identificó que para pacientes con infección confirmada por SARS COV2 en Nueva York, Estados Unidos, la mediana del tiempo de RCP fue de 14 minutos, $42 \%$ de los pacientes sobrevivieron a una primera parada cardíaca, sin embargo, ninguno sobrevivió al alta hospitalaria y el tiempo de supervivencia hasta la muerte fue de 2,8 horas (20). Mientras que en Wuhan, China, solo el $13,2 \%$ de los pacientes tuvieron RCE, el 2,94\% sobrevivieron durante al menos 30 días y un paciente logró un resultado neurológico favorable a los 30 días (21). En nuestra experiencia, el $58 \%$ de los pacientes han sobrevivido a una parada cardíaca, pero las tasas generales de supervivencia continúan siendo bajas. Hasta el momento no hay descritos en la literatura casos de fenómeno Lázaro en pacientes con COVID-19.

Finalmente, es importante que el personal de salud conozca el fenómeno de Lázaro. En ningún caso la muerte debe ser certificada tras el cese inmediato de la RCP. La necesidad de continuar la monitorización hemodinámica no invasiva por un tiempo razonable de al menos 10 minutos, si no es más, tras suspender la RCP es indispensable para comprobar la persistencia de asistolia previo a certificar la defunción; asimismo se debe corroborar la ausencia de funciones hemisféricas y del tallo cerebral. Algunos autores recomiendan, al ser la hiperinsuflación pulmonar una de las teorías más aceptadas y estudiadas de este fenómeno, desconectar el ventilador como último esfuerzo en pacientes que no responden a los esfuerzos de reanimación, sin embargo, queda a criterio del médico tratante (15).

CONTRIBUCIONES DE LOS AUTORES: concepción y diseño del estudio: AJOY, TRY, MCMA; recolección análisis e interpretación de datos: ADTS, MCMA; redacción del borrador del artículo: AJAH, TRY, ADTS; revisión crítica y aprobación de versión final: AJOY, CRDC; responsable de la veracidad e integridad del artículo: MCMA, AJAH.

CONFLICTOS DE INTERESES: Los autores declaran que no tienen conflictos de interés.

\section{REFERENCIAS}

1. Organización Mundial de la Salud. Enfermedad del coronavirus (COVID-19) Reporte de situación - 250. Abril 2021. Disponible en: https://www.who.int/docs/defaultsource/coronaviruse/situation-reports/20200816covid-19-sitrep-250.pdf?sfvrsn=5dde1 ca2 2

2. Sociedad Colombiana de Anestesiología y Reanimación: S.C.A.R.E. Manual de Práctica Clínica Basado en la Evidencia: Reanimación Cardiocerebropulmonar.1era ed. Bogotá, Colombia; 2014.

3. Interim Guidance for Basic and Advanced Life Support in Adults, Children, and Neonates With Suspected or Confirmed COVID-19. Circulation. 2020; 141: e933-e943. DOI: 10.1161/CIRCULATIONAHA.120.047463

4. Adhiyaman V, Adhiyaman S, Sundaram R. The Lazarus phenomenon. J R Soc Med. 2007; 100: 552-7.

5. Ministerio De Salud Y Protección Social. Orientaciones para el manejo, traslado y disposición final de cadáveres por SARS-COV-2 (COVID-19). 1era Ed. Bogotá, Colombia. 2020.

6. Bray JG. The Lazarus phenomenon revisited. Anesthesiology 1993; 78: 991. 
7. Gordon L, Pasquier M, Brugger H, Paal P. Autoresuscitation (Lazarus phenomenon) after termination of cardiopulmonary resuscitation - a scoping review. Scand J Trauma Resusc Emerg Med. 2020; 28(1): 14. Published 2020 Feb 26. doi:10.1186/s13049-019-0685-4

8. Hornby K, Hornby L, Shemie SD: A systematic review of autoresuscitation after cardiac arrest. Crit Care Med 2010, 38: 1246-1253.

9. Peña SB, Aedo IF, Palomino SL. Spontaneous return of circulation after termination of cardiopulmonary resuscitation maneuvers: a systematic review of cases of Lazarus phenomenon. Emergencias. 2014; 26: 307316

10. Huang et al.: Delayed recovery of spontaneous circulation following cessation of cardiopulmonary resuscitation in an older patient: a case report. Journal of Medical Case Reports 2013 7: 65.

11. Kämäräinen A, Virkkunen I, Holopainen L, Erkkilä EP, Yli-Hankala A, Tenhunen J. Spontaneous defibrillation after cessation of resuscitation in out-ofhospital cardiac arrest: a case of Lazarus phenomenon. Resuscitation. 2007; 75: 543-6.

12. Quick G, Bastani B. Prolonged asystolic hyperkalemic cardiac arrest with no neurologic sequelae. Ann Emerg Med. 1994; 24: 305-11.

13. Miller C. Towards evidence based emergency medicine: best BETs from the Manchester Royal Infirmary. BET 1: the use of adrenaline and long-term survival in cardiopulmonary resuscitation following cardiac arrest. Emerg Med J. 2013; 30: 249-50.
14. Sandroni C, Cavallaro F, Antonelli M. Is there still a place for vasopressors in the treatment of cardiac arrest? Crit Care. 2012; 16: 213.

15. Aufderheide TP, Lurie KG. Death by hyperventilation: a common and life-threatening problem during cardiopulmonary resuscitation. Crit Care Med. 2004; 32: S345-51.

16. Lapinsky SE, Leung RS. Auto-PEEP and electromechanical dissociation. N Engl J Med 1996; 335: 674 .

17. Figueroa Triana JF, et al. COVID-19 y enfermedad cardiovascular. Rev Colomb Cardiol. 2020. https://doi.org/10.1016/j.rccar.2020.04.004

18. D.B. Kramer, B. Lo, N.W. Dickert. CPR in the Covid-19 Era - An Ethical Framework. N Engl J Med 2020

19. National Academies of Sciences, Engineering, and Medicine. Rapid expert consultation on crisis standards of care for the COVID-19 pandemic. National Academies Press, 2020

20. Sheth V, Chishti I, Rothman A, Redlener M, Liang J, Pan D, Mathew J. Outcomes of in-hospital cardiac arrest in patients with COVID-19 in New York City. Resuscitation. 2020 Jul 21; 155: 3-5.

21. F. Shao, S. Xu, X. Ma, et al.In-hospital cardiac arrest outcomes among patients with COVID-19 pneumonia in Wuhan, China Resuscitation, 2020; 151: 18.23 